\title{
Visual and haptic perception of three-dimensional solid forms
}

\author{
CALVIN P. GARBIN and IRA H. BERNSTEIN \\ University of Texas, Arlington, Texas
}

\begin{abstract}
Subjects sorted 24 solid forms into groups on the basis of perceived similarity of shape, and rated each on a series of physical attributes under either visual or haptic conditions. Multidimensional scaling analyses within each condition produced highly similar two-dimensional solutions. Both first dimensions were related to size judgments, and both second dimensions were related to shape judgments. Correlations between haptic and visual attribute ratings were high, and the between-attribute correlational structures within each condition were also very similar. The findings are discussed in terms of Gibson's notion of partial perceptual invariances.
\end{abstract}

Brown and Owen and their associates have systematically explored Gibson's (1966) contention that the visual and haptic systems represent shape information in the same way. Brown and Owen (1967) produced 1,000 two-dimensional polygons using Attneave and Arnoult's (1956) Method I and analyzed 80 physical measures taken from each. Subsets of these forms provided well-defined shape forms for the study of visualhaptic equivalency in terms of complexity, size, and general shape perception.'

Brumaghim and Brown (1968) used an anchoring procedure to show that visual and haptic complexity ratings were highly related and that anchoring could occur in either cross-modal direction. Brown and Brumaghim (1968) also showed that multidimensional scaling (MDS) of "similarity of complexity" ratings for pairs of polygons produced a single dominant dimension (i.e., number of sides) for each modality. Finally, Owen and Brown (1970) reported that visual and haptic complexity ratings were highly correlated (.98) and strongly related to the number of sides of the polygon for both modalities.

Owen (1970) reported that visual and haptic size ratings were both associated with dispersion and elongation measures when polygon areas were held constant. However, size ratings from the two modalities were not as highly correlated (.68) as were complexity ratings, and only the haptic ratings correlated with number of sides. Thus, sensory equivalence was not as strongly supported for size perception as for complexity perception.

We would like to thank Donald L. Schurman both for providing the stimuli and for his comments about the design, James Gottlich for his help in collecting the data, Constance M. Pusen for her comments on an earlier version of this paper, and Martha Teghtsoonian for her considerable editorial efforts. Direct correspondence to Calvin P. Garbin, Psychology Department, University of Texas at Arlington, Box 19528, Arlington, TX 76019.
Owen and Brown (1970) found that response latencies for visual and haptic oddity and cross-modal match-tosample tasks each varied as similar quadratic functions of the number of sides of the polygon targets. They then employed multiple regression in an attempt to model the stimulus information used in these tasks. However, the results showed "few instances of common information utilization" (p. 305). Thus, although perceptual equivalence has been strongly supported for complexity information, and somewhat supported for size information, Gibson's notion has not received support from this series of studies in terms of the perception of complex holistic shape.

The present study compared visual and haptic perception, both of individual stimulus attributes and of holistic shape of irregular three-dimensional solid forms. Four interrelated hypotheses about perceptual equivalence were tested. The first hypothesis, that individual stimulus attributes are similarly perceived through the two modalities, was tested by correlating visual and haptic ratings of several attributes, including size and complexity. The second hypothesis, that the interrelationships among the various attributes are similarly perceived through the two modalities, was tested by comparing the factor structures of the attribute ratings from each modality. The third hypothesis, that visual and haptic perception of holistic shape (i.e., the perceptual structure) is similar, was tested by comparing the MDS solutions derived from shape similarity judgments under visual and haptic conditions (Isaac, 1970). The final hypothesis, that a similar set of attributes underlies both visual and haptic holistic shape perception (i.e., the definition of perceptual structure in each modality), was tested by comparing the stimulus attributes that describe the MDS solution space for each perceptual condition.

To provide a fair test of these hypotheses, each stimulus for this study had to have sufficient shape information and complexity to support, at least potentially, perceptions of "different shapes" for different 
subjects. That is, common geometric shapes would probably not be appropriate stimuli, because no one would be very surprised if visual and haptic subjects all formed separate homogeneous groups of circles, squares, stars, etc. The present three-dimensional stimuli seemed to fulfill these requirements at least as well as the polygons described above. Also, use of the present shapes would allow an examination of the generality of the findings using those polygons. This choice of stimuli introduced one substantial difference between the studies surveyed above and the present one: The former polygon forms were all quantified physically, whereas the present forms were quantified psychologically via subject ratings. Except for some simple attributes, for example, vertical extent, objective quantification of the present forms is substantially more difficult than it is for polygons. Thus, the use of these forms represents a tradeoff between quantifying forms objectively and broadening the range of complex forms used in the study of shape perception. Previous work (e.g., Monahan \& Lockhead, 1977) has shown that object classifications are better correlated with subjects' ratings than with physical measures. Therefore, use of psychological ratings of the attributes should not be construed as a major weakness of the present study.

\section{METHOD}

\section{Subjects}

One hundred right-handed undergraduates participated in this experiment to fulfill introductory psychology course requirements -50 subjects in each perceptual condition.

\section{Stimuli}

The 24 forms shown in Figure 1 were sampled randomly from a larger set of 45 stimuli that had been created to represent an informally defined population of complex irregular three-dimensional solid shapes. Each form was molded by hand from roughly the same volume of air-drying pottery clay. All forms could fit within a 3.5-in. sphere. Fingerprints and other fine texture features were removed prior to painting; the final

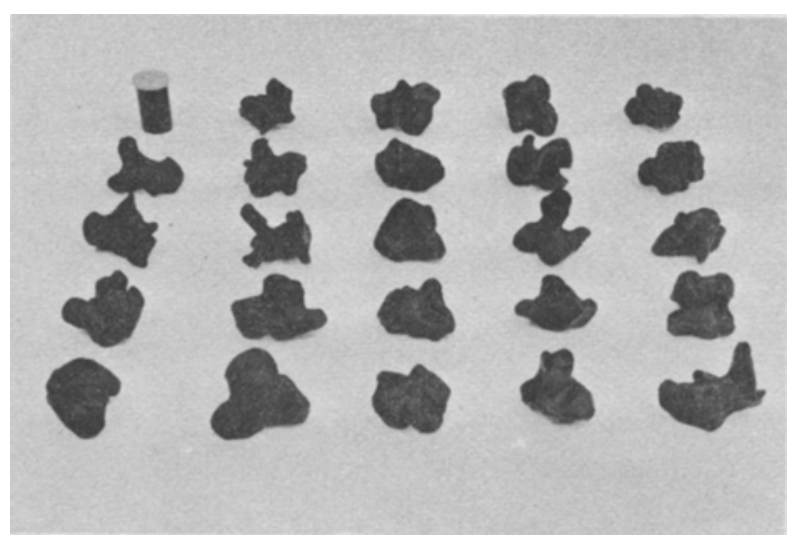

Figure 1. The 24 forms are shown along with a standard film canister for size comparison. Form number 1 is to the immediate right of the canister; forms are ordered from left to right and from back to front. painted weights ranged from 85 to $99 \mathrm{~g}$. Each form was assigned a base and remained resting on this base throughtout the procedure.

\section{Apparatus and Procedure}

The subjects were seated at a $60 \times 90 \mathrm{~cm}$ table with a neutral brown top. Each session started with the stimuli on their bases and arranged in four rows of six forms each. In the haptic condition, a sheet was draped from two poles reaching $60 \mathrm{~cm}$ above the tabletop and over the subject's shoulders to keep him or her from seeing the forms. In the visual condition, two small screens were used to keep the subject from seeing forms other than the one being judged.

All subjects sorted the 24 forms into groups, such that members of a group were similar in shape. The subjects were told that each form had a prescribed base that was to remain on the table top at all times, but that the orientation of the form on its base was not prescribed. That is, the subjects were told they were free to select any rotational orientation for each form, and they were encouraged to consider several such orientations before making their judgments. They were free to use any criteria or rules that they wished, except that the groups had to contain between two and eight forms. The subjects in the visual condition were told not to touch the forms except to rotate them on their bases or to slide them along the tabletop into groups. The subjects were watched carefully throughout the procedure to ensure that they did not explore the forms haptically. The subjects in the haptic condition were encouraged to use both hands to explore each form, but were not allowed to see the forms until after the sorting and rating procedures had been completed.

Following the sorting task, the subjects rated each form on each of 12 9-point bipolar attributes: (1) small vs. large, (2) short vs. tall, (3) narrow vs. wide, (4) dispersed vs. compact, (5) angular vs. round, (6) smooth vs. jagged features, (7) smooth vs. rough features, (8) simple vs. complex, (9) asymmetrical vs. symmetrical, (10) orientation not important vs. orientation important to shape, (11) shallow vs. deep, and (12) thin vs. thick.

The 12 attributes used in this study were those suggested by pilot subjects to describe the rules they had used to complete the sorting task. In addition, most of the attributes were related to measured or factored attributes used by Brown and his associates. Separation of the size measures short vs. tall and narrow vs. wide was possible because each form had a prescribed base.

The subjects were reminded that the rotational orientations at which the forms were presented were random and that they could orient the forms however they wished when making the various ratings. In addition, the meanings of the bipolar attributes were not, with two exceptions, specifically defined for the subjects. The subjects were asked to provide an "overall" rating of symmetry, rather than to select a single axis along which to give the rating. Also, the importance-of-orientation attribute was explained as the extent to which the form would change its overall shape if it were rotated on its base. These procedures were selected to explore the consistency with which the subjects applied the various attributes, particularly wide vs. narrow, shallow vs. deep, and thin vs. thick, all of which seemed to be "two-dimensional" judgments about the three-dimensional forms, which, unlike short vs. tall, could be applied to different aspects of the forms. Each subject rated the forms on the attributes in a different order.

There were no explicit time constraints for either the sorting or the rating task. Experimental sessions lasted about $55 \mathrm{~min}$ for visual sessions and $70 \mathrm{~min}$ for the haptic ones. All subjects completed first the sorting task and then the rating task. This ordering was chosen to ensure that subjects completed the sorting task using rules of their own design rather than adopting the stimulus attributes used in the rating task. 


\section{RESULTS}

\section{Analysis of Rating Data}

The rating data from the haptic and visual conditions were analyzed separately. Inspection of the resulting 288 frequency distributions and variances ( 24 forms $X$ 12 attributions) for each condition revealed that the subjects were in good agreement in their ratings, except for two of the three "two-dimensional" attributes mentioned just above, shallow vs. deep and thin vs. thick. Both of these produced nearly rectangular distributions across the 9-point scale and large variances for at least 20 of the 24 stimuli, for each perceptual condition. This indicated poor consistency in the application of these attributes across subjects. Consequently, these attributes were dropped from further analysis. The third "two-dimensional" attribute, narrow vs. wide, seemed to be consistently applied to the most narrow axis parallel to the tabletop. For the 10 attributes (excluding shallow vs. deep and thin vs. thick), bisquare estimates of central location were calculated for each attribute rating of each form.

The hypothesis that individual stimulus attributes are similarly perceived through the two modalities was tested by correlating visual and haptic ratings of each attribute. The leftmost column of Table 1 shows these correlations. In general, the visual and haptic ratings were highly correlated; in particular, small vs. large, short vs. tall, and simple vs. complex showed the highest correlations, in agreement with the work by Brown and his associates cited above.

The hypothesis that the interrelationships among the various physical attributes are similarly perceived through the two modalities was tested by examining how well the correlations among the attributes within each modality fit a common a priori factor structure. The physical attributes were hypothesized to represent three factors or categories: (1) size (small vs. large, short vs. tall, narrow vs. wide, and dispersed vs. compact), (2) shape (round vs. angular, smooth vs. jagged features, smooth vs. rough features, and simple vs. complex), and (3) symmetry (asymmetrical vs. symmetrical and importance of orientation).

The separation of size and shape attributes has been demonstrated both by Brown and Owen (1967) and by Richards (1972) in factor analysis of two-dimensional polygons. The decision to distinguish further between shape and symmetry measures was made on the basis of reports from pilot subjects and our own examination of the stimuli, and was given initial support by examinations of the correlations among the attributes. Various results from the research cited above and pilot work led to the hypothesis that there are substantial correlations between size and shape factors and between size and symmetry factors within each modality, and that there is a somewhat higher correlation between shape and symmetry factors.

The attribute ratings from each condition were submitted to separate oblique multiple groups (OMG) factor analyses to test this hypothesized three-factor structure and set of interfactor correlations. The oblique multiple groups factor analytic method was used because it, unlike the more common exploratory techniques (principal components and common factor with or without rotation), allows one to define factors in advance and then to test how well these a priori factors account for the data relative to alternative sets of factors. In addition, one can examine the interrelations among these defined factors and test hypotheses about these factor intercorrelations. The basic computations of an OMG analysis may be found in Harman (1976); for recent applications of this procedure see Bernstein and Eveland (1982), Bernstein, Garbin, and McClellan (1983), and Bernstein, Lin, and McClellan (1982).

The variance in the correlation matrix accounted for by the proposed structure was compared with that accounted for by other factor solutions that provided the best and worst fits for three factors. The amount of variance accounted for by three principal components (PC) is, by definition, the maximum amount possible.

Table 1

Correlations Between Visual and Haptic Attribute Ratings and OMG Structure Matrices and Interfactor Correlations

\begin{tabular}{|c|c|c|c|c|c|c|c|}
\hline \multirow[b]{2}{*}{ Attribute } & \multirow[b]{2}{*}{$\mathrm{r}$} & \multicolumn{3}{|c|}{ Visual Condition } & \multicolumn{3}{|c|}{ Haptic Condition } \\
\hline & & Size & Shape & Symmetry & Size & Shape & Symmetry \\
\hline Small-Large & .97 & $.96^{*}$ & -.39 & -.30 & $.97^{*}$ & -.29 & -.48 \\
\hline Short-Tall & .98 & $.88^{*}$ & -.62 & -.43 & $.86^{*}$ & -.54 & -.63 \\
\hline Dispersed-Compact & .85 & $-.74 *$ & .79 & .52 & $-.84 *$ & .70 & .70 \\
\hline Narrow-Wide & .88 & $.66^{*}$ & .09 & .09 & $.57 *$ & .29 & .21 \\
\hline Angular-Round & .73 & -.75 & $.88^{*}$ & .64 & -.31 & $.85^{*}$ & .48 \\
\hline Smooth-Jagged & .80 & .46 & $-.92 *$ & -.50 & .47 & $-.93 *$ & -.73 \\
\hline Rough-Smooth & .86 & -.26 & $.85^{*}$ & .34 & -.13 & .74 & .28 \\
\hline Simple-Complex & .94 & .44 & $-.96 *$ & -.72 & .42 & $-.93 *$ & -.73 \\
\hline Asymmetrical-Symmetrical & .81 & -.47 & .50 & $.91^{*}$ & -.48 & .68 & $.93 *$ \\
\hline Orientation Unimportant-Important & .63 & .20 & -.61 & $-.91 *$ & .44 & -.52 & $-.93 *$ \\
\hline Size Factor & & & -.53 & -.36 & & -.39 & -.50 \\
\hline Shape Factor & & & & .61 & & & .64 \\
\hline
\end{tabular}

*Indicates the a priori factor on which that attribute was hypothesized to load most heavily. 
Randomly formed three-factor solutions provided an estimate of the minimum amount of meaningful variance in the correlation matrix that could be accounted for by a three-factor model. These random models were formed by randomly choosing four attributes for the first factor, four for the second, and two for the third, without replacement. Thus, these factors mimicked the structure of the size-shape-symmetry model except for which attributes represented each factor.

A "good" proposed model will account for nearly as much variance as the PC model with the same number of factors, and substantially more variance than the corresponding random model(s). The proposed structure fit very well for both the visual and haptic data, in support of the second hypothesis. In the visual case, three PCs accounted for $85.9 \%$ of the variance $(\lambda=5.76$, 1.84 , and .99), the proposed structure accounted for $85.0 \%$ of the variance, and the average variance accounted for by the three random models was $60.4 \%$ for the haptic data, three PCs accounted for $85.3 \%$ of the variance $(\lambda=5.58,1.96$, and 1.00$)$, the proposed model accounted for $82.5 \%$ of the variance, and the average variance accounted for by the three random models was 63.2\%. The OMG structure matrices and the interfactor correlations are shown in Table 1.

An examination of the structure matrices shows that interrelations among the attributes were very similar for the haptic and visual conditions. Each attribute has a higher loading on the proposed factor (indicated by an asterisk) than on the other factors, except in one case. The attribute dispersed vs. compact is about equally correlated with both the size and shape factors in the visual condition, but not in the haptic condition. As hypothesized, the shape and symmetry factors are more highly correlated than are the other combinations, for both conditions. The factors are significantly correlated, in both conditions ( $p<.05$ with $\mathrm{df}=22$ ), indicating that, for these stimuli, the size, shape, and symmetry scales are not perceived independently of one another.

\section{Analysis of Sorting Data}

Subjects in the haptic condition tended to form slightly fewer groups than those in the visual condition [means $=5.12$ and $5.77, t(98)=2.31, p<.05]$. Thirtyseven haptic subjects formed four or five groups, whereas only 25 visual subjects did. Twelve haptic subjects formed more than five groups, whereas 25 visual subjects did so.

Individual sorting performances were represented in the off-diagonals of a $24 \times 24$ dissimilarity matrix in which a 0 indicated that the item pair had been grouped together and a 1 meant that the pair had not been grouped together. Data from subjects in the visual and haptic conditions were analyzed separately, using the SAS (Young \& Lewyckyj, 1979) version of ALSCAL. Separate individual differences analyses were carried out under assumptions that the data were: (1) interval level, (2) ordinal level from a discrete underlying pro- cess, and (3) ordinal level from a continuous underlying process. To help exclude the possibility that solutions were the result of local minima, multiple analyses with different starting configurations were carried out for each of the three sets of data assumptions.

The various ordinal/discrete analyses did not converge to very similar solutions or to similar goodness-of-fit values, and will not be discussed further. Interval and ordinal/continuous assumptions led to very similar solutions and to similar goodness-of-fit values for the various analyses. The remaining discussion will be in terms of the ordinal/continuous solutions.

Solutions in two through six dimensions were obtained for each condition; ALSCAL does not provide one-dimensional individual differences type of solutions. The values presented are from solutions for which the algorithm chose the starting configuration and are representative of the other solutions. For the visual data, the average Kruskal Formula 1 stress values for two through six dimensions were $.138, .134, .131, .129$, and .124 , and the average R-square values for the 50 subjects were $.923, .935, .939, .942$, and .945 . For the haptic data, the average stress values were $.169, .143, .136$, .132 , and .129 , and the average R-square values were $.878, .879, .882, .885$, and .887 .

For both the haptic and visual data, the two-dimensional solutions fit the sorting data well, and there was no apparent advantage to the more complex solutions. Additional analyses were carried out to determine whether a one-dimensional solution might be sufficient. Visual and haptic composite matrices were formed by summing corresponding elements of the 50 individual matrices within each condition. Each composite matrix was analyzed separately using ALSCAL. The three data assumptions and multiple starting configurations were used with results very similar to those reported above. The results presented are from the ordinal/continuous analyses for which the algorithm selected the starting configuration.

For the visual data, the stress values for one through three dimensions were $.172, .093$, and .086 and the R-square values were $.823, .942$, and .963 . For the haptic data, the stress values were .194, .112, and .099 and the R-square values were $.744, .912$, and .921 . Thus, it seems that, for both the visual and the haptic data, the second dimension adds substantially to the model, but the third dimension does not. ${ }^{2}$

Figures 2 and 3 , respectively, show the visual and haptic individual differences solutions in two dimensions. The two solutions are generally similar, although some elements are located differently. For example, the stimulus groups $(4,7,9,17,22),(11,12,21,24)$, and $(6,10,13,14,16)$ appear in both solutions and in nearly the same relative positions. The groups are more tightly formed in the visual solution, and form 19 is included in the first group in the haptic solution. Inspection of the raw sorting data indicated that these groups (or their proper subgroups, e.g., $4,7,9$, or $11,12,24$, etc.) 


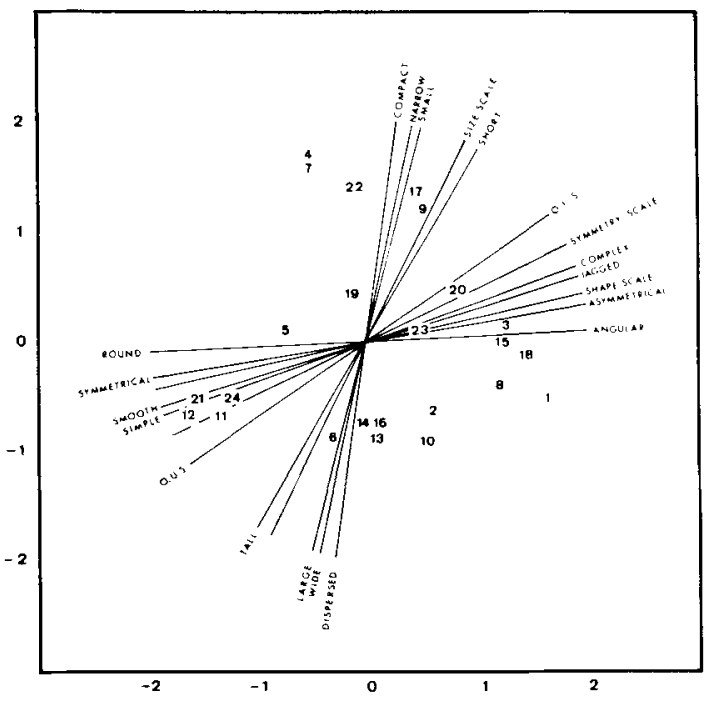

Figure 2. Two-dimensional visual MDS solution; Dimension I is the vertical axis. (O.I.S. = orientation important to shape; O.U.S. = orientation unimportant to shape.)

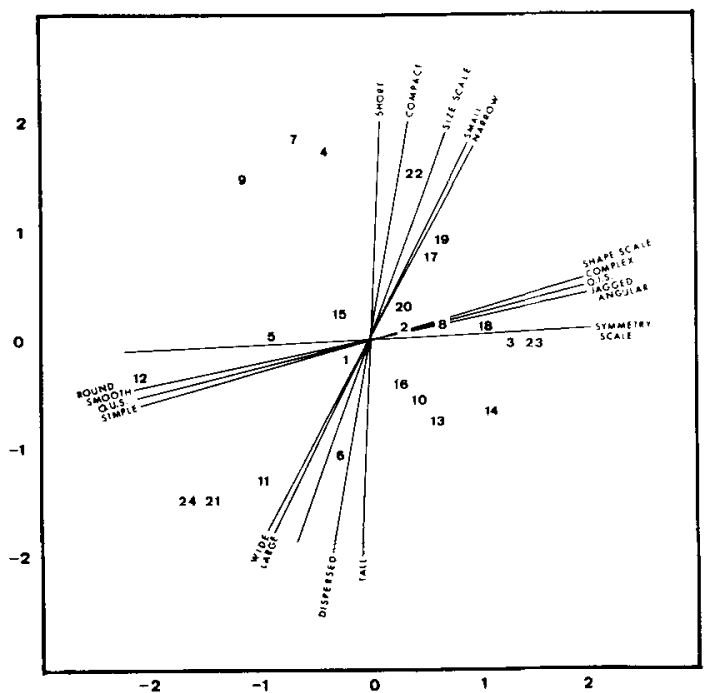

Figure 3. Two-dimensional haptic MDS solution; Dimension 1 is the vertical axis. (O.I.S. = orientation important to shape; O.U.S. = orientation unimportant to shape.)

appear more frequently in the visual than in the haptic condition. Thus, the tighter clusters of the visual solutions reflect greater between-subject consistency in that condition than in the haptic condition. The slightly higher R-square values for the visual than for the haptic MDS solutions reflect this consistency also. Certain forms do have substantially different positions in the two solutions. As one example, forms 1 and 15 and, to a lesser extent, form 2 are located near form 18 in the visual solution but near form 5 in the haptic solution.

The third hypothesis, that visual and haptic perceptions of holistic shape are similar, was tested by comparing the visual and haptic MDS solutions. The extent of their similarity was measured by the coefficient of congruence (CC) (Wrigley \& Neuhaus, 1955, cited in Harman, 1976). These CC values were .90 for Dimension I and .73 for Dimension II.

Cross-validation analyses within each condition provided the basis for evaluating these results. The 50 subjects in each perceptual condition were randomly split into two groups of 25 subjects each. Separate individual differences analyses were carried out for the four sets of data; the data were treated as ordinal/continuous, and the algorithm selected the starting configuration. For the visual condition, the CC values for Dimensions I and II were .95 and .88 ; for the haptic data, they were .93 and .78 . Thus, the $\mathrm{CC}$ of .90 reported above indicates that Dimension I of the haptic solution is very similar to Dimension I of the visual solution, whereas the corresponding Dimension IIs $(\mathrm{CC}=.73)$ are less similar. In addition, the second dimension within each condition is somewhat less stable than the first, and this is more true of the haptic data.

The fourth hypothesis, that visual and haptic judgments of holistic shape are based on a similar set of stimulus attributes, was tested in terms of the attributes that best describe the MDS solutions from each condition. A multiple regression procedure was used to interpret the two-dimensional MDS solution from each condition in terms of the 10 subjective attribute ratings and three attribute scales. The attribute scales correspond to the size, shape, and symmetry factors discussed above, and were formed as the sum of the appropriate attribute ratings. ${ }^{3}$ In each of 13 analyses within each condition, one of the attributes or an attribute scale was regressed over the coordinates of the MDS solution. The inverse cosines of the normalized regression beta weights from each model were used to define the vector in the MDS solution that corresponded to that attribute or attribute scale (Kruskal \& Wish, 1978). Table 2 shows the results from the analysis of each attribute and of the size, shape, and symmetry scales.

Table 2

Multiple Correlations Between Attribute Ratings and MDS Solution Coordinates

\begin{tabular}{lll} 
& Visual & Haptic \\
\hline Small vs. Large & $.86^{* *}$ & $.78^{* *}$ \\
Short vs. Tall & $.86^{* *}$ & $.91^{* *}$ \\
Dispersed vs. Compact & $.61^{*}$ & .57 \\
Narrow vs. Wide & $.80^{* *}$ & $.90^{* *}$ \\
Angular vs. Round & $.87^{* *}$ & $.63^{*}$ \\
Smooth vs. Jagged Features & $.69^{*}$ & $.80^{* *}$ \\
Rough vs. Smooth Features & .41 & .26 \\
Simple vs. Complex & $.80^{* *}$ & $.72^{* *}$ \\
Asymmetrical vs. Symmetrical & $.71^{*}$ & .58 \\
Unimportant vs. Important & $.79^{* *}$ & $.67^{*}$ \\
Size Scale & $.92^{* *}$ & $.88^{* *}$ \\
Shape Scale & $.76^{* *}$ & $.72^{* *}$ \\
Symmetry Scale & $.80^{* *}$ & $.66^{*}$ \\
\hline
\end{tabular}

${ }^{*} p<.01 . \quad{ }^{* *} p<.001$. 
Figures 2 and 3 show the vectors of those attributes and scales that passed a goodness-of-fit criterion of $\mathrm{p}<.01$ (Kruskal \& Wish, 1978).

Dimension I, the dominant dimension in both solutions, is a size information dimension for both. The size scale vector is similarly located in both solutions, at $64 \mathrm{deg}$ in the visual solution and at $69 \mathrm{deg}$ in the haptic solution. The individual size attributes, however, are somewhat differently positioned. For example, tall vs. short is the one most aligned with Dimension $I$ in the haptic solution but the one least aligned in the visual solution. Note that although the attribute dispersed vs. compact weighed about equally on the size and shape factors in the visual case, as shown in Table 1, it clearly lies with the other attributes of the size factor in the visual MDS solution. For this reason, dispersed vs. compact was included in the size scale for the visual solution, as it was for the haptic solution.

Dimension II is a shape/symmetry information dimension in both solutions. The various shape and symmetry attribute vectors are more highly aligned in the haptic than in the visual solution; also, the relative positions of the symmetry and shape scale vectors are reversed in the two solutions, although in general the vectors of the individual shape and symmetry attributes are intermixed.

Individual differences analyses provide information concerning how each subject employed the solution dimensions. This information is given by ALSCAL as the proportion of variance of each subject's data that was accounted for by the dimensions of the MDS solution, thus showing the relative importance of each dimension. This information was explored in two ways. Cluster analysis (Howard, 1977) of these data revealed no distinct groups of subjects. Analysis of angular variation (Schiffman, Reynolds, \& Young, 1981) revealed no consistent gender differences for either perceptual mode. Most subjects weighted the first dimension of the solutions more heavily than the second in their sorting patterns, as would be expected.

\section{DISCUSSION}

Results from each of the four phases of the analysis give support for Gibson's claim of perceptual equivalence between the visual and haptic systems. Visual and haptic ratings of physical attributes tend to be strongly correlated, and the interrelations among these attributes are highly similar for the two senses. Sorting procedures produced two-dimensional MDS solutions that were highly similar, especially along the dominant axis (size attributes). Finally, interpretation of the MDS solution in terms of the rated attributes indicated that subjects in the two conditions used similar rules to sort the forms.

Although the bulk of the present evidence supports Gibson's claim, differences in the visual and haptic MDS solutions and their interpretations were found that are predominantly related to the Dimension IIs of the solutions. One of these is that the various shape and symmetry attribute vectors were more highly aligned with each other in the haptic than in the visual solution. This suggests that less distinction was made among these attributes under the haptic condition than under the visual condition. Perhaps greater differentiation among these shape and symmetry attributes would occur if the haptic subjects were more familiar with the forms. Alternatively, perhaps these attributes are more highly correlated when shapes are perceived haptically. The visual-haptic literature provides little help on this issue. While visual discrimination is typically found to be better than haptic discrimination (see Jones, 1981, and Marks, 1978, for excellent reviews), and haptic discrimination is known to improve with practice (Gibson, 1966; Simons \& Locher, 1979), these findings all refer to discriminations among stimuli rather than among stimulus attributes, an important distinction. Thus, the present finding is of a somewhat different sort from those previously reported, and it remains to be seen whether this apparent visual-haptic difference can be attributed to some form of familiarity or practice effect.

In the visual solution, the shape scale is more aligned with Dimension II than is the symmetry scale, whereas the converse is true in the haptic solution. Closer inspection of the figures reveals that the shape scale vector is similarly located in each solution (13 deg for visual, $16 \mathrm{deg}$ for haptic), whereas the position of the symmetry vector differs substantially. The third difference between visual and haptic solutions also mainly involves the Dimension IIs. As noted above, some forms are in different relative positions in the two solutions. These differences most often involve displacement along Dimension II. This was also reflected in the different $\mathrm{CC}$ values comparing visual and haptic solutions for Dimension I (.90) and Dimension II (.73). In addition, haptic Dimension II was somewhat less stable when cross-validated than was that dimension of the visual data (CC values .78 and .88 , respectively). As with the lesser discrimination among the shape and symmetry attributes in the haptic case mentioned above, this may be an effect of unfamiliarity with the stimuli. In this case, however, findings such as those by Gibson (1966) and Simons and Locher (1979) are of direct value, and based upon them, one might expect greater visual-haptic similarity with additional stimulus familiarity.

In this study, subjects were permitted to form as many groups, of whatever size, as they chose (within the limits of two to eight forms per group), and usually formed four to six groups. An alternative procedure would be to direct subjects to form groups of various sizes on different trials (Harvey \& Gervais, 1978), say of two, three, four, six, eight, and twelve forms. Data from this procedure would indicate whether visual and haptic structures become more or less similar when subjects are required to make discriminations that are more coarse or more fine than those they normally would 
make. To have used this procedure in the present study would have invited an unwelcome complication. A subject might "change rules" as s/he was asked to create different sorting patterns, so that a single analysis based on these sorts would confound rules and produce an MDS solution that represented none of them.

All of the studies discussed, including this one, have looked at form perception in terms of various continuous metric attributes of the forms. That is, they have ignored distinguishing characteristics, or substructures of the forms. For example, forms 11,13 , and 16 all have a vertical projection that many subjects referred to as a "handle," and forms 4 and 8 both had "places to fit your fingers between the ridges." Similar reports were found during informal investigations with polygons similar to those used by Richards (1972). Subjects seem to have sorted items in terms of attributes rather than characteristics, even though they reported noticing these characteristics, were free to use any sorting rules they liked, and had not yet been introduced to the attributes used in the study. For example, Figures 2 and 3 show that forms 4 and 8 are in separate clusters.

In conclusion, this study lends support to Gibson's claim of supramodal perceptual invariances, but suggests that the invariants for irregular three-dimensional solid forms may be at the level of composites such as size and shape, rather than at the level of specific attributes or distinguishing substructures.

\section{REFERENCES}

Atrneave, F., \& Arnoult, M. D. (1956). The quantitative study of shape and pattern perception. Psychological Bulletin, 53, 452-471.

Bernstein, I. H., \& Eveland, D. C. (1982). State vs. trait anxiety: A case study in confirmatory factor analysis. Personality \& Individual Differences, 3, 361-372.

Bernstein, I. H., Garbin, C. P., \& McClellan, P. G. (1983). A confirmatory factoring of the California Psychological Inventory. Educational and Psychological Measurement, 43, 687-692.

Bernstein, I. H., Lin, T. D., \& McClellan, P. G. (1982). Cross- vs. within-racial judgments of attractiveness. Perception \& Psychophysics, 32, 495-503.

Brown, D. R., \& Brumaghin, S. H. (1968). Perceptual equivalence, pattern perception and multidimensional methods. Perception \& Psychophysics, 4, 253-256.

Brown, D. R., \& OWEN, D. H. (1967). The metrics of visual form: Methodological dyspepsia. Psychological Bulletin, 68, 243-259.

Brumaghim, S. H., \& Brown, D. R. (1968). Perceptual equivalence between visual and tactual pattern perception: An anchoring study. Perception \& Psychophysics, 4, 175-179.

GiBson, J. J. (1966). The senses considered as perceptual systems. Boston: Houghton Mifflin.
Harman, H. H. (1976). Modern factor analysis (3rd ed.). Chicago: University of Chicago Press.

Harvey, L. O., Jr., \& Gervais, M. J. (1978). Visual texture perception and Fourier analysis. Perception \& Psychophysics, 24, 534-542.

How ARD, J. H., JR. (1977). More on the statistical treatment of individual differences in multidimensional scaling. Perception \& Psychophysics, 22, 106-107.

IsAAC, P. D. (1970). Dissimilarities as indices of individual perceptual structure. Perception \& Psychophysics, 7, 229-233.

JoNES, B. (1981). The developmental significance of cross-modal matching. In R. D. Walk \& H. L. Pick, Jr. (Eds.), Intersensory perception and sensory integration. New York: Plenum Press.

KRUSKAL, J. B., \& WISH, M. (1978). Multidimensional scaling. Beverly Hills: Sage Publications.

MARKs, L. E. (1978). The unity of the senses: Interrelationships among the modalities. New York: Academic Press.

Monahan, J. S., \& Lockhead, G. R., (1977). Identification of integral stimuli. Journal of Experimental Psychology: General, 106, 94-110.

Nunnally, J. C. (1978). Psychometric theory (2nd ed.). New York: McGraw-Hill.

OWEN, D. H. (1970). Shape correlates of visual and tactual size judgments: A contrast with complexity. Perception \& Psychophysics, 8, 20-22.

Owen, D. H., \& Brown, D. R. (1970). Visual and tactual form discrimination: Psychophysical comparison within and between modalities. Perception \& Psychophysics, 7, 302-306.

RICHARDs, C. G. (1972). A multidimensional scaling analysis of judged similarity of complex forms in two task situations. Perception \& Psychophysics, 12, 154-160.

Schiffman, S. S., Reynolds, M. L., \& Young, F. W. (1981). Introduction to multidimensional scaling: Theory, methods, and application. New York: Academic Press.

Simons, R. W., \& Locher, P. J. (1979). Role of extended perceptual experience upon haptic perception of nonrepresentational shapes. Perceptual and Motor Skills, 48, 987-991.

Young, F. W., \& LEWYCKYJ, R. (1979). ALSCAL 4: Users guide. (Available from F. W. Young, Department of Psychology, University of North Carolina, Chapel Hill, NC 27514.)

\section{NOTES}

1. In this research, the touch condition was referred to as tactual. Because of the abundance of vibrotactual research that has appeared recently, the distinguishing label "haptic" will be used here.

2. These two-dimensional solutions were very similar to the corresponding individual differences solutions. Individual differences scaling was used to exclude the possibility that solutions based on aggregated data might not represent individuals' data.

3. These were unweighted sums. Scales formed as proper OMG component scores and as weighted sums were both correlated above .90 with these unweighted sums, and the corresponding vectors in the MDS solutions differed by no more than 2 deg. This robustness of unit weighted scales is commonly found (e.g., Nunnally, 1978).

(Manuscript received October 17, 1983; revision accepted for publication June 5, 1984.) 